\title{
Variability due to hospital on mortality in oncological gastric surgery in Spain
}

\author{
Touma-Fernandez A. ${ }^{1}$, Cura-Iglesias S. ${ }^{1}$, Castillo-Bustos J. ${ }^{1}$, Perez-Lopez M. ${ }^{2}$, Baré M. ${ }^{3}$, \\ Sarriá-Santamera A. ${ }^{4}$
}

${ }^{1}$ Hospital Universitario Morales Meseguer, Department of Anaesthesiology, Murcia, Spain, ${ }^{2}$ Universidad de Alcala, Department of Surgery, Madrid, Spain, ${ }^{3}$ Universidad Autónoma de Barcelona, Epidemiología Clínica y Cribado de Cáncer, Barcelona, Spain, ${ }^{4}$ Instituto de Salud Carlos III, Agencia de Evaluación de Tecnologías Sanitarias, Madrid, Spain.

Background and Goal of Study: High attributable variability to hospital on outcomes, if present, may indicate a substantial room of improvement in health care delivery. This study aims to quantify the variation in risk adjusted mortality in oncological gastric surgery between hospitals.

Materials and methods: A retrospective national population based study using data from "Conjunto Mínimo de Datos al Alta Hospitalaria" (CMBD) of the "Sistema Nacional de Salud" (SNS) in Spain was performed. All SNS patients discharged with diagnosis of gastric cancer (ICD-9 codes 151.XX and 230.2) that underwent total or partial gastrectomy (ICD codes 43.XX) between 1 January 2006 and 31 December 2009 were identified, and a random intercept logistic regression model for hospital cluster was fitted, achieving risk adjustment through variable model selection using two standard criteria, namely the loglikelihood and discrimination of the model using the area under the receiver operating characteristics (AUROC) curves. Once the final model being fitted, to assess the amount of variation between hospitals, the random effects variance was calculated, and the intraclass correlation coefficient (ICC) roughly interpreted as the proportion of total variation explained by clustering, was also determined.

Results and discussion: We identified 7,547 patients who had oncological gastric surgery in one of 248 hospitals, with a raw mortality rate of $8.77 \%$. The risk adjustment model included age (odds ratio [OR] 1.07, $95 \%$ confidence interval $[\mathrm{Cl}]=1.06$ to $1.08, \mathrm{p}<$ 0.001 ), low mortality ICD-9 diagnosis -(diagnosis codes were amalgamated in low and high mortality due to the great number of codes to account for)-, (OR $0.59,95 \% \mathrm{Cl}=0.49$ to $0.71, \mathrm{p}<0.001)$, charlson score greater than zero (OR $1.43,95 \% \mathrm{Cl}=1.20$ to $1.71, \mathrm{p}<0.001$ ), female sex (OR $0.76,95 \% \mathrm{Cl}=0.64$ to $0.91, p=0.003$ ), and elective surgery (OR 0.46 ,
$95 \% \mathrm{Cl}=0.39$ to 0.56$)$. The model had an AUROC curve of $0.76(95 \% \mathrm{Cl}=0.73$ to 0.77$)$ indicating good discrimination ability. Patient related factors accounted for $91.8 \%$ of the outcome variation, with a considerable impact of hospital on outcome (ICC of $8.2 \%)$.

For the sake of comparison, the discriminative ability of the standard and the random intercept logistic regression model was also compared, with a clear improvement of discriminative ability in the model that includes the cluster effect as shown in Figure 1.

\section{ROC Curve}

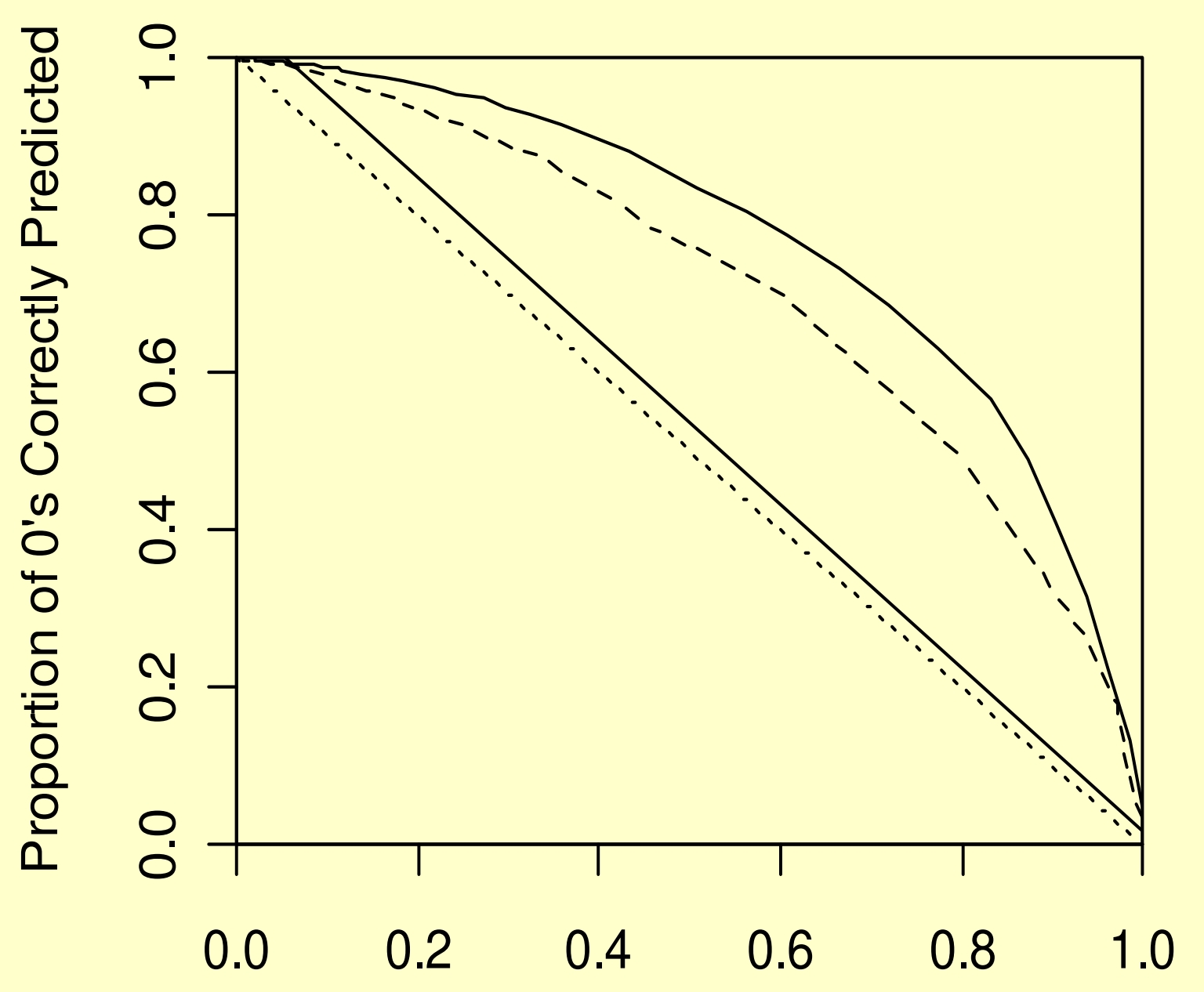

Figure 1. Proportion of deaths correctly predicted with standard (dashed line) and random intercept logistic model (continue).

Limitations: Like in most of the observational studies, it cannot be ruled out the presence of a constant risk fallacy, case mix fallacy and overfitting. We do not know to which extent results could be biased due to these limitations.

Conclusion: Most of the outcome variability in oncological gastric surgery is related to patients factors, however, a considerable amount is related to hospital factors indicating a room for improvement. 